\title{
Research on Infiltration Mathematics Thought during the Classroom Teaching \\ Yao Tong
}

Jinzhou Experimental School, Jinzhou, China

2749241144@qq.com

Keywords: Classroom teaching; Mathematics; Activity inquiry; Infiltration thought

\begin{abstract}
Mathematics is root is mathematical thinking. The primary of student mathematics study is to master and apply the mathematics' idea. When the student goes into society, the mathematics knowledge point may be forgotten. However the mathematics mind and thought will be left deeply in the mind. All this is valuable for the youngster, and bring far reaching influence to the future work and learning. Therefore, thought infiltration in mathematics teaching is essential and important. Guide students to feel mathematics idea during the exploration discovery, guide students to comprehend mathematics idea during the discussion conclusion and guide students to apply mathematics idea during the create applications. The purpose is to let the mathematics idea into student brain, which would provide good foundation for students in the future work and study.
\end{abstract}

\section{Acknowledgement}

This research was financially supported by the 2014 distinguished teacher workshop of Liaoning Province.

\section{课堂教学中渗透数学思想研究}

\author{
佟瑶 \\ 实验学校, 中国 辽宁省 锦州 121000 \\ $2749241144 @ q q . c o m$
}

摘要: 数学的根就是数学思想。学生数学学习的根本就是掌握、应用数学的思想。学生在进 入社会后, 所学的数学知识也许会遗忘但是那种铭刻于头脑中的数学精神和数学思想方法, 对于学生来说是弥足珍贵的, 对学生未来的工作和学习将产生深远的影响。因此在教学中渗 透数学思想是非常必要和重要的。可以在在探究发现中引导学生感受数学思想, 在讨论归纳 中引导学生领悟数学思想, 在创造应用中引导学生应用数学思想, 使数学思想根植于学生的 大脑，为孩子将来的工作和学习奠定良好的基础。

关键词: 课堂教学1; 数学2; 活动探究式3; 渗透思想4

\section{前言}

著名学者弗里德曼 [1]说: “数学逻辑结构的一个特殊的和最重要的要素就是数学思想, 整个 数学学科就是建立在这些思想的基础上, 并按照这些思想发展起来的。” 这句话明确的告诉我 们, 数学的根就是——数学思想。由此我们可以确定, 学生数学学习的根本就是掌握、应用 数学的思想。只有根植在数学思想基础上的数学学习才是有效的, 高效的 $[2-5]$ 。正如日本著 名数学教育家米山国藏 [6]教授所说: “学生在学校所学的数学知识, 在进入社会后, 几乎没 有机会应用, 因而这种作为知识的数学, 通常在出校门后不到一两年就忘掉, 然而不管他们 从事什么工作, 那种铭刻于头脑中的数学精神和数学思想方法, 却长期地在他们的生活和工 作中发挥着重要作用, 使其终身受益”。这一论断的阐述印证了数学思想在学习与生活中的 
重要意义 [7-10]。数学思想在学习中有如此重要的意义, 我们在平时的教学中进行了一些有 价值的尝试。

\section{1. 在探究发现中引导学生感受数学思想}

探究发现是 “活动探究式” 教学模式的中心环节, 重点强调 “做中学”, 主要是教师在提供 素材的基础上, 启发学生动手操作, 自主探究。在这一环节中, 我们鼓励学生操作实践, 主 动探究, 引导学生在操作探究中主动发现知识背后蕴含的数学思想。我们都知道, 数学思想 与数学知识不同, 它隐含于知识的发生、发展和应用过程中, 并与概念的抽象与概括、公式 的推导与建立、规律的发现与归纳以及问题的分析与解决过程密切相关、彼此交融。数学思 想的体现要以知识为载体, 通过探究发现让其根植于学生的头脑, 逐步发展成为一种意识、 观念和素养。在教学中, 要合理地把学生熟悉的、了解的、感兴趣的数学事例搬进课堂, 在 对实际问题数学化的过程中, 让学生经历探究过程, 感受其中蕴含的数学思想。

下面就以两位数加一位数的进位加法为例, 说一说在探究活动中如何引导学生感受数学思想。 在教学时, 教者出示例题 $28+4$, 启发学生自己探索计算的方法。在自主探究学习中, 有的孩 子借助计数器计算, 有的孩子使用摆小棒的方法数出结果, 有的孩子把 4 分成 2 和 2 , 先用 $28+2=30$, 再用 $30+2=32$, 有的孩子把 28 分成 20 和 8 , 先算 $8+4=12$, 再算 $20+12=32$, 还有的 孩子采用了列坚式计算的方法。以上学生采用的方法, 除了列坚式计算是本课要学习的新知 识, 其他方法都是以前学习过的旧知识来解决新问题。借助学具、摆小棒计算出结果的孩子, 他们采用的是数形结合的方法, 使用拆数计算方法的孩子, 他们的做法是, 化难为易, 化未 知为已知。虽然, 孩子们在解决问题的过程中已经很好的体现了数学思想的应用, 但是这种 应用是模糊的, 朦胧的, 盲目的。他们的头脑中, 还没有形成明确的、有目的的应用数学思 想解决问题的意识, 这时就需要教师的引领。所以, 在教学进行到这个环节的时候, 教者向 学生提出了一个问题, 请你将黑板上的五种方法分分类。起初, 孩子们分成了两大类, 一类 是摆学具的方法, 一类是计算的方法。经过进一步的观察, 又将计算方法细化, 一类是用旧 知识解决问题, 一类是用新知识解决问题。抓住这一契机, 教者及时小结, 从而让同学们体 会到, 能够化繁为简, 化未知为已知, 这样的方法可以帮助我们解决问题, 我们可以在以后 的学习中应用这种方法解题。通过问题分类和小结, 在学生的头脑中初步渗透了数形结合和 化归的数学思想。

这种感受和体会在对于学生来说是弥足珍贵的, 不仅对学生现在的学习具有辅助和促进作用, 在学生未来的工作和学习也将产生深远的影响。

\section{2. 在讨论归纳中引导学生领悟数学思想}

讨论归纳也是 “活动探究式” 教学模式的中心环节。重点体现 “生生对话”, 教师把学生不同 的思维方式和相关信息 “搜集上来”, 组织对话互动, 对自己和他人的观点进行思辨, 从而在 交流中内化、形成自己的观点, 最终使问题得到解决, 实现师生共享。在这一环节, 以生生 交流和师生交流的方式, 将知识的探讨向纵深方向发展, 引导学生透过表象看本质, 感悟数 学知识背后的数学思想。

比如, 在学习加法的认识一课时, 我进行了这样一个活动: 在黑板上贴出 3 个苹果, 接着又 出示两个苹果, 引导学生观察并用自己的话说出, 刚才老师做了什么? 学生总结出: 有 3 个 苹果, 又拿来 2 个苹果, 一共有 5 个苹果。在此基础上, 提出问题, 像这样的事情你会做吗? 请你动手摆一摆。学生有的摆小正方体, 有的摆圆形, 有的摆三角形, 还有的用拨计数器的 方法来表示。在充分展示汇报之后, 将举例的范围拓展, 引导学生思考, 在生活中, 你还遇 到过这样的事情吗? 学生纷纷举出实例, 有 4 只小狗, 又跑来 5 只, 一共是 9 只; 妈妈吃了 1 个苹果, 我吃了 1 个苹果, 一共吃掉 2 个苹果; 树上有 2 只小鸟, 又飞来 3 只, 现在有 5 只小鸟; 河里游来 1 群小鱼, 后来又游来 2 群小鱼, 现在河里有 3 群小鱼…… 
在列举了大量实例的基础上, 我提出了本节课的关键问题, 刚才我们举的例子有一个共同点, 是什么你知道吗? 经过短暂的思考, 孩子们想到了, 列举的实例都是在原来的基础上数量增 加了。那么像这样的问题我们可以用什么数学方法解决呢? 当提出这个核心问题时, 学生水 到渠成的想到了加法。

上面列举的教学实例从学生已有的生活经验出发, 通过观察、操作、归纳、概括的环节, 引 导学生在大量的生活实例中剥离出具有价值的数学方法, 体会问题最本质的属性, 感悟加法 的意义, 从而明白了在什么情况下要用加法来解决问题。这样的学习过程, 不仅自然而然地 渗透了数学模型思想而且培养了学生建构模型的能力, 提高学生解决问题的能力, 进而使学 生获得对数学理解的同时, 在思维能力、情感态度与价值观等多方面得到发展。”

又如, 用字母表示数在讨论归纳环节, 学生领悟到符号化思想给学习带来的直观性、间接性、 科学性。三角形三边关系一课的学习, 在讨论第三条边的取值范围时, 学生领悟到了极限思 想。对于例子、习题, 不要就题论题, 要引导学生学会反思, 可以用这样的问题: 你是怎样 想出来的? 关键是哪一步? 回顾探究过程; 你更喜欢哪种方法? 这个方法好在哪? 优化方法, 感悟数学思想; 通过解决这个题, 我们应该学什么? 培养学生应用数学思想方法的意识, 提 升学生数学素养。著名数学教育家弗赖登塔尔指出: 反思是数学活动的核心和动力。让学生 养成回顾、反思的习惯, 这种反思能较好地概括思维本质, 从而上升到数学思想方法上来。

\section{3. 在创造应用中引导学生应用数学思想}

创造应用是 “活动探究式” 教学模式的最后一个环节。此环节教师要组织学生将探索归纳出 的新知识、新方法用于实践, 学会应用知识解决问题。这一环节是引导学生在形成技能的基 础上向能力方向转化, 提高学生运用知识解决实际问题的能力。教师不仅要培养学生应用知 识的能力, 更要帮助学生树立应用数学思想解决同类问题的意识, 为学生解决类似问题广开 思路。例如在教学《6的乘法口诀》时, 董笑老师学生在完成想一想、算一算的练习中, 先让 学生计算, 再通过交流自己的算法, 以 “ $7 \times 6+6$ ” 为例, 借助图片用课件演示来理解算式的 意义, 运用数形结合启发将算式转化为 $8 \times 6$ 来计算, 渗透转化的数学思想, 懂得两个算式形 式虽不同, 表示的意义以及结果是相同的。又如让学生算一算每个图中各有多少个格子, 教 师启发学生怎样将其他图形转化成与第一个图形相同的排列方法, 可以直接用口诀计算? 学 生通过实际操作, 动手剪一剪、拼一拼, 转化成长方形后分别用 $6 \times 3 、 4 \times 3$ 来计算, 从而感 受到化繁为简数学思想的魅力。

在创造应用环节充分发挥数学思想对发现解题途径的定向、联想和转化功能, 举一反三, 触 类旁通。使学生形成自觉地运用数学的思维方式去思考和处理现实问题的习惯, 就实现了数 学学习的价值。

\section{4. 致谢}

感谢辽宁省名师工作室大力支持。

\section{参考文献}

[1] 李志强. 探究式教学的探索与实践. 当代教育论坛：学科教育研究, Vol.4(2006) No.4, p.69-70.

[2] 吴绪玫. 谈科学课程探究式教学的困难与对策. 云南教育, Vol.35(2003) No.5, p.29-31.

[3] 王丹. 试论探究式教学的特点及教师的作用. 教育学报, Vol.10(1998) No.7, p.21-24.

[4] 戴志聪, 杜道林. 论高等教育中以学生为主体的教学模式的构建. 中共山西省直机关党校 学报, Vol.5(2015) No.5, p.30-31. 
[5] 郑艈信. 学习理论的现代发展及其教学涵义. 数学教育学报, Vol.13(2004), No.1, p. 10-16.

[6] 王丹, 吴孟达, 毛紫阳. 数学建模课程教学的定位与思考. 高等教育研究学报, Vol.38(2015), No.1, p.116-120.

[7] 间洁. 数学方法论中 “化归法” 的教学反思. 数学学习与研究: 教研版, Vol.2(2015) No.2, p.5-5.

[8] 周锡花. 渗透思想方法完成探究教学——浅谈小学数学开展探究式教学. 启迪与智慧: 教 育版 (中), Vol.18(2015) No.6, p.82-82.

[9] 蔡丹, 文茗, 周璇. 基于认知加工过程的数学训练方案述评. 心理科学, Vol.38(2015) No.5, p.1116-1122.

[10]张昆, 曹一鸣. 完善数学教师教学行为的实现途径. 数学教育学报, Vol.1(2015) No. 1, p.008.

\section{References}

[1] Li Zhiqiang. Research-style teaching exploration and practice. Contemporary education BBS: subject education research, Vol.4 (2006) No.4, p.69-70.

[2] Wu Xumei. Talk about difficulty and countermeasure of science curriculum. Yunnan education, Vol.35 (2003) No.5, p.29-31.

[3] Wang Dan. Talk about the characteristics of inquiry teaching, and the teacher function. Journal of Educational Studies, Vol.10 (1998) No.7, p.21-24.

[4] Dai Zhichong, Du Daolin. Theory of higher education to students as the main body of the construction of the teaching mode. The communist party of China (Shanxi made authority party school journal, Vol.5 (2015) No.5, p.30-31.

[5] Zheng Yumin. The modern development of learning theory and its teaching meaning. Journal of Mathematics Education, Vol.13 (2004), No.1, p. 10-16.

[6] Wang Dan, Wu Mengda and Mao Zhiyang. Thinking and orientation for the teaching of mathematical modeling course. Uournal of Higher Education Research, Vol.38 (2015), No.1, p.116-120.

[7] Yan Jie. Teaching reflection of mathematical methodology in "transforming method". Mathematics study and research, Vol.2 (2015) No.2, p.5-5.

[8] Zhou Xihua. Penetrating thought method to complete the inquiry teaching. Inspiration and wisdom: Education edition, Vol.18 (2015) No.6, p.82-82.

[9] Cai Dan, Wen Ming and Zhou Xuan. Based on the cognitive process of mathematical training program review. Psychological science, Vol.38 (2015) No.5, p.1116-1122.

[10]Zhang Kun, Cao Yiming. Approach of the improve the mathematics teacher's teaching behavior. Journal of Mathematics Education, Vol.1 (2015) No. 1, p.008.

作者简介: 佟瑶 $(1976-)$, 女, 辽宁省葫芦岛, 小学高级, 主要研究方向为小学数学教育 教学实践研究, E-mai1：2749241144@qq.com。 